\title{
Two-Photon Coherent Polarization Flipping of Confined Excitons
}

\author{
P. MaChNiKOWsKI \\ Institute of Physics, Wrocław University of Technology \\ Wybrzeże Wyspiańskiego 27, 50-370 Wrocław, Poland
}

\begin{abstract}
A two-photon process leading to coherent transitions between the two circularly polarized exciton states in a quantum dot is studied. It is shown that optical flipping of the exciton polarization is possible with picosecond laser pulses. The process is closely related to two-photon Rabi oscillations of a biexciton but it is much more stable against shifts of the laser frequency.
\end{abstract}

PACS numbers: 78.67.Hc

\section{Introduction}

Recent years have witnessed enormous progress in the optical control of charge states in semiconductor quantum dots (QDs). For instance, coherent optical transitions (Rabi oscillations) between the ground state and a single-exciton state of a single QD have been demonstrated [1]. This and other experiments show that quantum optical schemes can be implemented on these artificial systems. Another experimental achievement in this field is the observation of two-photon Rabi oscillations between the ground and biexciton states of a single self-assembled QD [2]. This experiment has shown that coherent control of two-photon transitions in QDs far beyond the perturbative regime is feasible.

On the other hand, the dynamically developing field of spintronics and spin-based implementations of classical and quantum information processing has motivated the search for fast, optical methods of control for confined electron spins [3-6]. Experiments in this area [7, 8], even though still limited to QD ensembles, seem very promising.

This paper extends the theory of two-photon optical control of a biexciton system [2] and shows that two-photon transitions may be used to coherently rotate the spin orientation (polarization) of a single exciton confined in a QD. 


\section{Theory and results}

Let us consider a single QD coupled to a linearly polarized laser beam. According to the selection rules, such a beam couples both the exciton states to the ground and biexciton states. We assume that the frequency of the beam is detuned from both these transitions, as shown in Fig. 1a. For pulses of picosecond durations the excited states of confined carriers are irrelevant and may be disregarded. We will also neglect the exchange interaction that couples the two circularly polarized exciton states and turns them into a weakly split linearly polarized doublet [9]. The energy of this coupling is usually below $100 \mu \mathrm{eV}$ and does not affect the system dynamics on the timescales relevant for the present discussion.
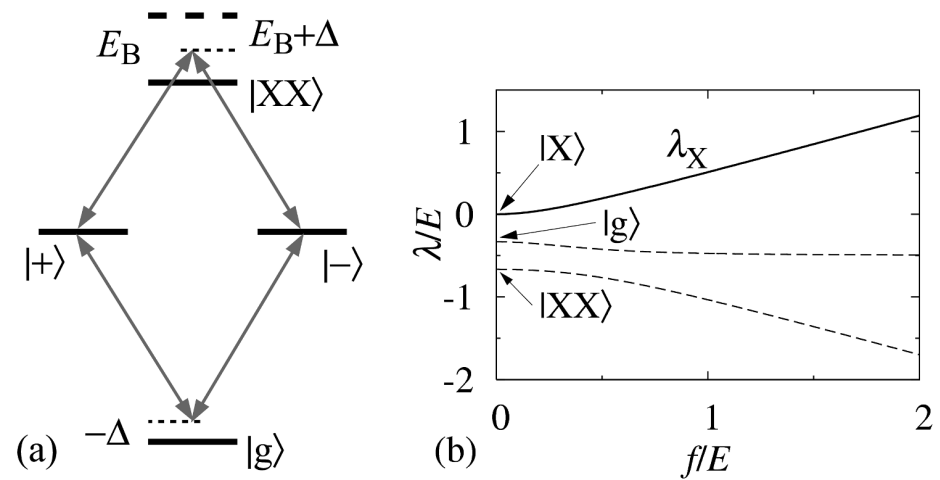

Fig. 1. (a) The energy level structure of a confined biexciton and the schematic presentation of the optical couplings between the states. (b) The spectral branches representing the instantaneous eigenstates of the Hamiltonian as a function of the pulse amplitude $f$ (the adiabatic parameter). Here $\Delta=-E_{\mathrm{B}} / 3, E_{\mathrm{B}}>0$.

Thus, in the rotating wave approximation the system is described by the Hamiltonian

$$
H=-\left(E_{\mathrm{B}}+\Delta\right)|\mathrm{XX}\rangle\langle\mathrm{XX}|+\Delta| g\rangle\langle g|+\frac{1}{2} f(t)[(|g\rangle+|\mathrm{XX}\rangle)\langle\mathrm{X}|+\text { h.c. }]
$$

where $|g\rangle,|\mathrm{XX}\rangle$ denote the ground and biexciton states, $|\mathrm{X}\rangle=(|+\rangle+|-\rangle) / \sqrt{2}$ is the bright (" $x$-polarized") superposition of the two single-exciton states $|+\rangle,|-\rangle$ with definite circular polarizations, $E_{\mathrm{B}}$ is the biexciton shift (assumed positive in the binding case, as in Fig. 1), $\Delta=\omega-E$ is the detuning between the laser frequency $\omega$ and the single-exciton transition energy $E$, and $f(t)$ is the envelope of the laser pulse amplitude. The other, dark (" $y$-polarized") superposition of the exciton states, $|Y\rangle=(|+\rangle-|-\rangle) / \sqrt{2}$, remains decoupled.

We will assume that the system is initially prepared in one of the single-exciton states, say $|+\rangle$. This state can be represented as $|+\rangle=(|\mathrm{X}\rangle+|\mathrm{Y}\rangle) / \sqrt{2}$. The state $|\mathrm{Y}\rangle$ is decoupled from the laser beam and does not evolve. The evolution of the state $|\mathrm{X}\rangle$ under appropriate conditions may be found using the adiabatic 
theorem $[10,2]$. To this end, let us consider the diagram of instantaneous eigenstates of the relevant three-level Hamiltonian (excluding the decoupled state $|-\rangle$ ) as a function of the pulse amplitude $f$, shown in Fig. 1b. As can be seen, in general the spectral branch containing the state $|\mathrm{X}\rangle$ remains non-degenerate and separated from the other states by at least $\min \left(|\Delta|,\left|\Delta+E_{\mathrm{B}}\right|\right)$. Here, we will only consider the situation when the detunings of the laser frequency from both transition energies, $|\Delta|$ and $\left|\Delta+E_{\mathrm{B}}\right|$, are much larger that both the maximum pulse amplitude and the pulse spectral width. In such case, the state $|+\rangle$ undergoes an adiabatic evolution, with $f(t)$ playing the role of a slowly varying parameter. After the pulse has been switched off, the initial state is restored with the additional dynamical phase

$$
\alpha=-\frac{1}{\hbar} \int_{-\infty}^{\infty} \mathrm{dt} \lambda_{\mathrm{X}}(t)
$$

where $\lambda_{\mathrm{X}}(t)$ is the instantaneous eigenstate of the Hamiltonian (1) corresponding to the state $|\mathrm{X}\rangle$ (see Fig. 1b). As a result, the state $|+\rangle$ undergoes the transformation

$$
|+\rangle=\frac{|\mathrm{X}\rangle+|\mathrm{Y}\rangle}{\sqrt{2}} \longrightarrow \frac{\mathrm{e}^{\mathrm{i} \alpha}|\mathrm{X}\rangle+|\mathrm{Y}\rangle}{\sqrt{2}}=\mathrm{e}^{\mathrm{i} \alpha / 2}\left(\cos \frac{\alpha}{2}|+\rangle+\mathrm{i} \sin \frac{\alpha}{2}|-\rangle\right) .
$$

The phase $\alpha$ may be arbitrarily large. Moreover, for a fixed pulse shape it is a monotonous function of the pulse intensity. Thus, by varying the pulse amplitude, the exciton can be coherently rotated between the two polarization states $|+\rangle$ and $|-\rangle$, much like in the usual pulse-area dependent Rabi oscillation between the ground and single exciton states, induced by a resonant circularly polarized beam [1].

The instantaneous eigenvalue $\lambda_{\mathrm{X}}(t)$ results from diagonalizing a three-level Hamiltonian which can always be done analytically. However, the resulting formula is reasonably simple only in the symmetrically detuned case $\Delta=-E_{\mathrm{B}} / 2$, when one finds

$$
\lambda_{\mathrm{X}}(t)=E_{\mathrm{B}} \frac{\sqrt{1+8\left[f(t) / E_{\mathrm{B}}\right]^{2}}-1}{4} .
$$

In the general case, it seems more reasonable to calculate the system evolution numerically.

The final occupation of the $|-\rangle$ state as a function of the pulse area

$$
\theta=\int_{-\infty}^{\infty} \mathrm{d} t f(t)
$$

for various detuning conditions is shown in Fig. 2a. In all the cases, the exciton undergoes oscillations between the two polarization states. In spite of some qualitative similarity to the Rabi oscillations in a two-level system (described by the universal function $\sin ^{2} \theta / 2$ ) one can clearly see essential differences. The oscillations are not strictly periodic, especially for weak pulses, where the transition probability develops very slowly. In fact, for weak pulses the occupation of the 

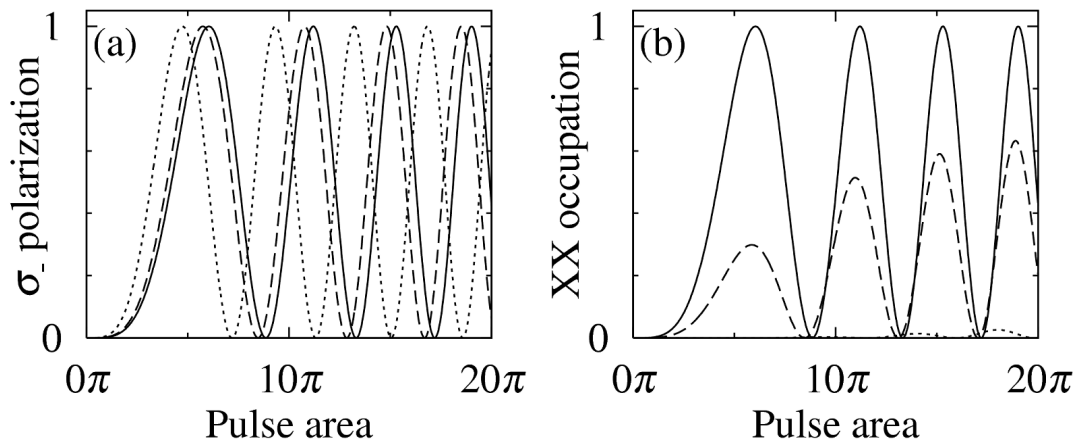

Fig. 2. (a) Two-photon polarization flip: the occupation of the state $|-\rangle\left(\sigma_{-}\right.$-polarized exciton) as a function of the pulse area, for $\tau_{0}=5 \mathrm{ps}, E_{\mathrm{B}}=4 \mathrm{meV}$, and $\Delta=E_{\mathrm{B}} / 2$ (solid), $E_{\mathrm{B}} / 3$ (dashed), and $E_{\mathrm{B}} / 6$ (dotted). (b) Two-photon Rabi oscillations [2]: the occupation of the biexciton state as a function of the pulse area, for $\tau_{0}=5 \mathrm{ps}, E_{\mathrm{B}}=$ $4 \mathrm{meV}$, and $\Delta=E_{\mathrm{B}} / 2=2 \mathrm{meV}$ (solid), $\Delta=1.9 \mathrm{meV}$ (dashed), and $\Delta=1.7 \mathrm{meV}$ (dotted).

flipped state grows as $\theta^{4} \sim I^{2}$, where $I$ is the pulse intensity, as expected for a two-photon process (see Ref. [2]). Moreover, it is clear from Eq. (2) that the rotation angle is a nonlinear functional of the pulse envelope. Hence, contrary to the usual Rabi oscillations, no universal area theorem exists for the final occupations.

Let us note that the symmetrical detuning condition is equivalent to the two-photon resonance between the ground and biexciton states. Thus, in this case the two-photon polarization flip results from the same evolution as the two-photon Rabi oscillations of the biexciton [2] (only the initial state is different). The very good agreement between the description based on the adiabatic theorem and the experimental results [2] shows that the theory captures the essentials of the quantum evolution under actual laboratory conditions (except for dephasing effects that are obviously beyond the scope of the present theory).

There is, however, a fundamental difference between the two-photon biexciton oscillations and the polarization flip. If the two-photon resonance condition $\Delta=E / 2$ is not satisfied, the transition from the ground to biexciton state is forbidden and the corresponding Rabi oscillations are precluded. On the contrary, the polarization flip is always resonant. For comparison of the two processes the results for two-photon Rabi oscillations are plotted in Fig. 2b. While the curves describing the two processes at the two-photon resonance (symmetric detuning) are the same, they become very different out of resonance. This additional freedom of control may be useful for optimizing experimental parameters against phonon-induced dephasing $[11,12]$.

The two-photon polarization flip described here is expected to take place under the same conditions as the two-photon Rabi oscillations that have already been demonstrated in an experiment [2]. Moreover, the polarization of an exciton 
is relatively easily measurable. Therefore, it should be possible to observe the two-photon polarization flip using currently available experimental techniques. This would provide an additional degree of control over the biexciton system which can be treated as a two-bit quantum register if the logical values of 0 and 1 are associated with the absence and presence, respectively, of an exciton with one of the two circular polarizations. Like two-photon Rabi oscillations, the two-photon polarization flip is an entangling operation, with maximally entangled states of the two excitons, of the form $(|01\rangle \pm \mathrm{i}|10\rangle) / \sqrt{2}$, reached for $\alpha=(n+1 / 2) \pi$.

\section{References}

[1] A. Zrenner, E. Beham, S. Stufler, F. Findeis, M. Bichler, G. Abstreiter, Nature 418, 612 (2002).

[2] S. Stufler, P. Machnikowski, P. Ester, M. Bichler, V.M. Axt, T. Kuhn, A. Zrenner, Phys. Rev. B 73, 125304 (2006).

[3] F. Troiani, E. Molinari, U. Hohenester, Phys. Rev. Lett. 90, 206802 (2003).

[4] Pochung Chen, C. Piermarocchi, L.J. Sham, D. Gammon, D.G. Steel, Phys. Rev. $B$ 69, 075320 (2004).

[5] A. Nazir, B.W. Lovett, S.D. Barrett, T.P. Spiller, G.A.D. Briggs, Phys. Rev. Lett. 93, 150502 (2004).

[6] B.W. Lovett, A. Nazir, E. Pazy, S.D. Barrett, T.P. Spiller, G.A.D. Briggs, Phys. Rev. B 72, 115324 (2005).

[7] M.V.G. Dutt, Jun-Cheng, B. Li, X. Xu, X. Li, P.R. Berman, D.G. Steel, A.S. Bracker, D. Gammon, S.E. Economou, Ren-Bao-Liu, L.J. Sham, Phys. Rev. Lett. 94, 227403 (2005).

[8] A. Greilich, R. Oulton, E.A. Zhukov, I.A. Yugova, D.R. Yakovlev, M. Bayer, A. Shabaev, A.L. Efros, V. Merkulov, V. Stavarache, D. Reuter, A. Wieck, Phys. Rev. Lett. 96, 227401 (2006).

[9] E.L. Ivchenko, Phys. Status Solidi A 164, 487 (1997).

[10] A. Messiah, Quantum Mechanics, North-Holland, Amsterdam 1966.

[11] A. Krügel, V.M. Axt, T. Kuhn, P. Machnikowski, A. Vagov, Appl. Phys. B 81, 897 (2005).

[12] K. Roszak, A. Grodecka, P. Machnikowski, T. Kuhn, Phys. Rev. B 71, 195333 (2005). 